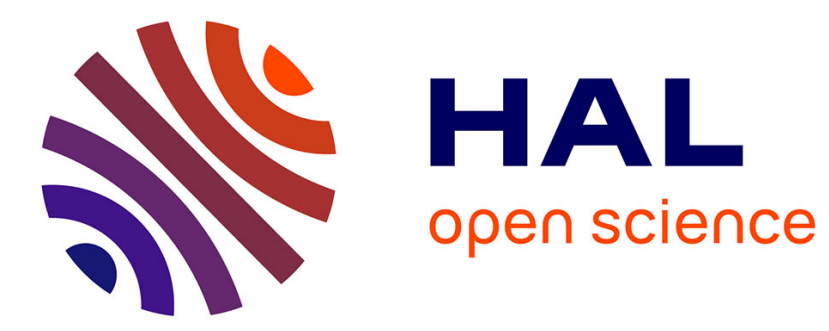

\title{
Morphological Characterization of Graphene Plans Stacking
}

\author{
Albane Borocco, Clémentine Fellah, James Braun, Marie-Hélène Berger, Petr \\ Dokládal
}

\section{- To cite this version:}

Albane Borocco, Clémentine Fellah, James Braun, Marie-Hélène Berger, Petr Dokládal. Morphological Characterization of Graphene Plans Stacking. ISMM 2017, May 2017, Fontainebleau, France. pp.435446, 10.1007/978-3-319-57240-6_35. hal-01482431

\section{HAL Id: hal-01482431}

https://hal-mines-paristech.archives-ouvertes.fr/hal-01482431

Submitted on 3 Mar 2017

HAL is a multi-disciplinary open access archive for the deposit and dissemination of scientific research documents, whether they are published or not. The documents may come from teaching and research institutions in France or abroad, or from public or private research centers.
L'archive ouverte pluridisciplinaire HAL, est destinée au dépôt et à la diffusion de documents scientifiques de niveau recherche, publiés ou non, émanant des établissements d'enseignement et de recherche français ou étrangers, des laboratoires publics ou privés. 


\title{
Morphological Characterization of Graphene Plans Stacking
}

\author{
Albane Borocco ${ }^{1}$, Clémentine Fellah ${ }^{2,3}$, James Braun ${ }^{2}$, Marie-Hélène Berger ${ }^{3}$, and Petr \\ Dokládal ${ }^{1}$ \\ 1 MINES ParisTech, PSL Research University, CMM - Centre for Mathematical Morphology, 35, rue St. \\ Honoré, 77305 Fontainebleau, France, email: firstname.lastname@mines-paristech.fr \\ 2 CEA, DEN, SRMA, LTMEX, F-91191 Gif-sur-Yvette, France, email: firstname.lastname@cea.fr \\ 3 MINES ParisTech, PSL* Research University, CMAT - Centre for Materials, France, email: \\ firstname.lastname@mines-paristech.fr
}

\begin{abstract}
The graphene is a material obtained when carbon atoms form large planar molecules. Well organized, large graphene molecules stacked ontop each other convey to graphene particularly interesting properties useful in nuclear industry.

Understanding how the organization on the molecular scale influences the mechanical properties of the material is a key element in the material manufacturing process. In this scope, features like local orientation and length have already been largely explored in the literature. This paper brings a new feature evaluating the number of plans stacked ontop each other and the length of this stacking. It allows obtaing other features such as the overall rate of organization or locality and preferential orientation. These informations, synthesized in the form of histograms provides a key information in the processus the material design.

Experimental results obtained on images taken by an electronic scanning microscope are presented to illustrate the proposed method.
\end{abstract}

Keywords: Mathematical Morphology, Orientation space, Filtering, Graphene.

\section{Introduction}

Ceramic matrix composite materials are candidates for thermostructural applications in extreme environments such as in turbojet engines or core materials for future nuclear reactors $[1,6]$. Foremost among them stand $\mathrm{SiC} / \mathrm{SiC}$ composites which are composed of a $\mathrm{SiC}$ matrix by high purity silicon carbide $(\mathrm{SiC})$ fibers reinforced. This combination of two refractory, brittle components gives rise to a damage tolerant material, thanks to a pyrocarbon $(\mathrm{PyC})$ interphase deposited between the fibers and the matrix to control the fiber/matrix bonding strength [9].This interphase and their related interfaces allow crack deflection and crack bridging by the fibers during mechanical loading which is the key to achieve a pseudo-ductile macroscopic behavior. Optimization of their properties through the process must be performed and are from far a big concern. A thorough investigation of the carbon rich fiber surface structure and of the nature of the fiber/ $\mathrm{PyC}$ interphase bonding is essential to control and improve the fiber/matrix coupling in $\mathrm{SiC} / \mathrm{SiC}$ composites [2].

A variety of techniques could be used to characterize the carbon nanostructure of the $\mathrm{SiC} / \mathrm{SiC}$ composite interface including Raman spectroscopy, X-ray Diffraction (XRD) and Transmission Electron Microscopy (TEM). The dark field and high-resolution modes in transmission electron microscopy allow imaging directly 002 lattice fringes of graphene layers and to identify their morphological, textural and structural parameters. But most of the information obtained from TEM images have been qualitative or semi-quantitative [12].The direct interpretation of the TEM features is not easy considering contrast variations, layers twisting and overlapping and local changes in the organization of carbon nanostructure.

Image analysis allows eliminating problems of representativeness for the observations of graphene layers nanostructure and more quantitative data can be obtained $[15,13,18]$.Various parameters of the spatial arrangement of carbon lamellae can be measured such as lattice fringe length, size and shape, curvature and tortuosity of plans. A lot of studies are already published in the literature studying on the nanostructure of fibers, soot, carbon black, pyrolytic carbon, fullerenes 
and coal chars by image processing $[5,11,7,4,19,16]$. In this study, the proposed technique based on mathematical morphology involves the characterization of quantitative indices for describing the carbon interface of $\mathrm{SiC} / \mathrm{SiC}$ composite. This new method leads rapidly to the detection of graphene layers stacking thanks to the development of a more important robustness to noise and to quantify especially their local and space orientations using HRTEM images. The final goal is to establish the local interaction mechanisms of carbon phases at the interface and the mechanisms that control mechanical behavior of $\mathrm{SiC} / \mathrm{SiC}$ composites. Thereafter it will be possible to define optimized materials with these information for high-temperature applications.

\section{Detection of graphene plans and their local orientation}

This section explains how to detect graphene plans and their orientation by modeling each plan by a centered, threadlike curve of same length and orientation.

\subsection{Thresholding}

The High-resolution transmission electron microscopy (HRTEM) allows to image individual graphene molecules (in bright) and the gaps in between (that appear darker). A thresholding may dissociate the structures from the background, see Fig. 1(a). The Otsu's method [10] is thresholding algorithms that iterates over all possible threshold values and calculates the spread of the pixel levels each side to find the optimum.

The Otsu's threshold is the value minimizing the sum of foreground and background spreads, defined as a weighted sum of variances of the two classes:

$$
t=\arg \left(\min _{t}\left(\sigma_{W}^{2}(t)\right)\right), \text { with } \sigma_{W}^{2}(t)=\omega_{1}(t) \sigma_{1}^{2}(t)+\omega_{2}(t) \sigma_{2}^{2}(t)
$$

with $\sigma_{i}^{2}$ being the variance of the class $i$ and $\omega_{i}$ the weight given by its likelihood.

As the material may contain globally darker and brighter regions, a global threshold fails locally, Fig. 1(b). Instead, the threshold should be calculated locally: for each pixel, an optimal threshold $\mathrm{t}$ is determined in the local neighborhood defined by a sliding window. Its size is fixed so as to contain several graphene plans and gaps, but small enough not to contain both a bright and a dark region. We have fixed the widow size to five times the plan width. Fig. 1(c) illustrates the result of a local Otsu thresholding. It allows the extraction of the graphene plans despite local intensity variation. Let BW denote the binary image representing the graphene plans in the original grayscale image I.

$$
B W(x, y)=\left\{\begin{array}{l}
1, \text { if } I(x, y)>t(x, y) \\
0, \text { otherwise }
\end{array}\right.
$$

\subsection{Skeletonization}

In order to model each plan by a centered threadlike curve with the same length, the skeleton is extracted from the binary image. This operation uses an algorithm [20] that removes the points of the outline of the shape while preserving its topological characteristics. Let Sk denote from now the skeleton.

$$
S k: I \rightarrow \text { skeleton }(B W)
$$

The skeleton is then pruned to remove spurious edges. The pruning is done using the algorithm 1 that allows to remove spikes shorter than a certain size.

By applying this algorithm, spikes shorter than $n$ are removed. However, isolated structures shorter than $n$ are preserved, and the connectivity is not modified. A convenient size for $n$ is roughly equivalent to the interreticular distance. Fig. 2 illustrates the impact of pruning on the skeleton. 


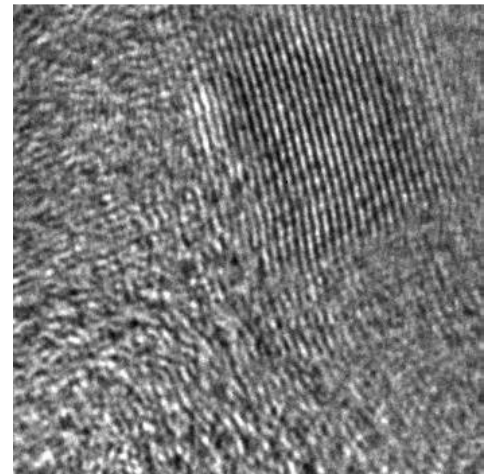

(a) Initial image

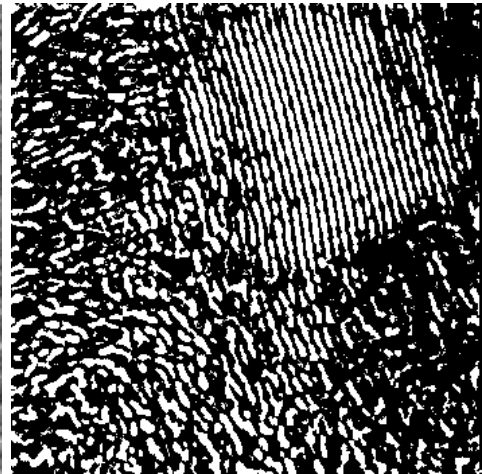

(b) global threshold

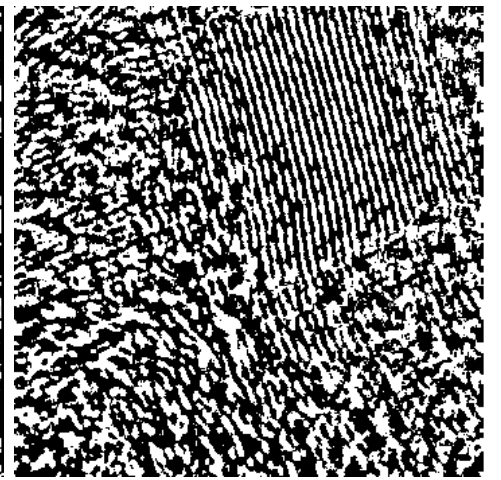

(c) local threshold

Fig. 1. Application of Otsu's threshold. (a) initial image. (b) global Otsu thresholding. (c) sliding-window Otsu thresholding.

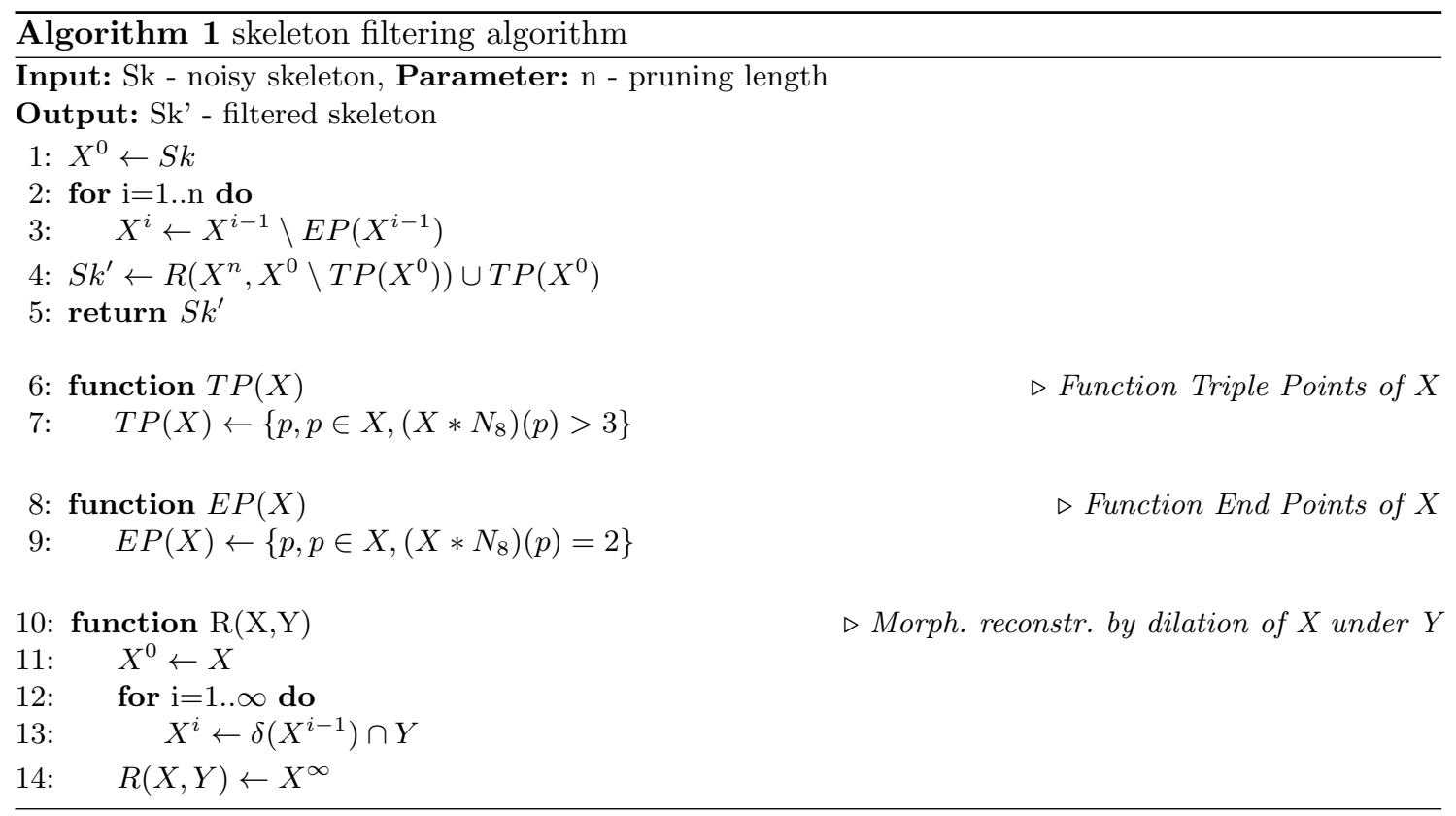

\subsection{Local orientation}

In order to determine the local orientation of each graphene plan, we first compute the local orientation of each pixel. This calculation is based on the morphological opening: For each discrete angle value $\theta$, we compute the opening of the grayscale image by $B_{\theta}$, a segment tilted by angle $\theta$. The local orientation of a pixel is the one that maximizes the opening value.

$$
\mid \begin{aligned}
\theta: \quad D & \rightarrow] 0, \pi] \\
(x, y) & \mapsto \arg \left(\bigvee_{\theta \in] 0, \pi]}\left(\gamma_{B_{\theta}}(I)(x, y)\right)\right)
\end{aligned}
$$

In the case of multiple maximum values, the local orientation resulting is the first maximum encountered, the process to cope with that loss of information is explained in section 3 The local orientation of each graphene plan is obtained by combining the local orientation of the gray scale image and the skeleton of the binary image.

Fig. 3 illustrates (in false colours) the results of local orientation on the gray-scaled image (a) and on the skeleton (b). 


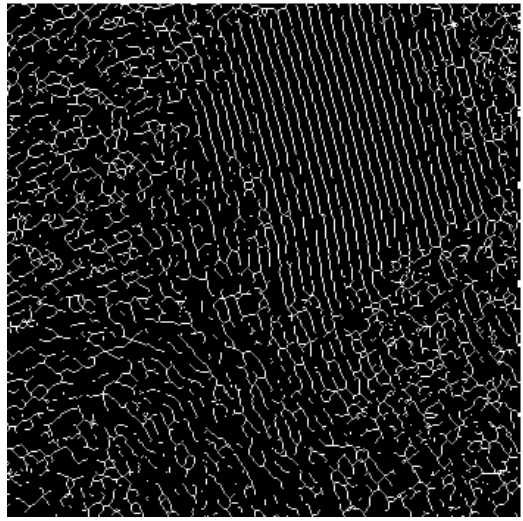

(a) Skeleton

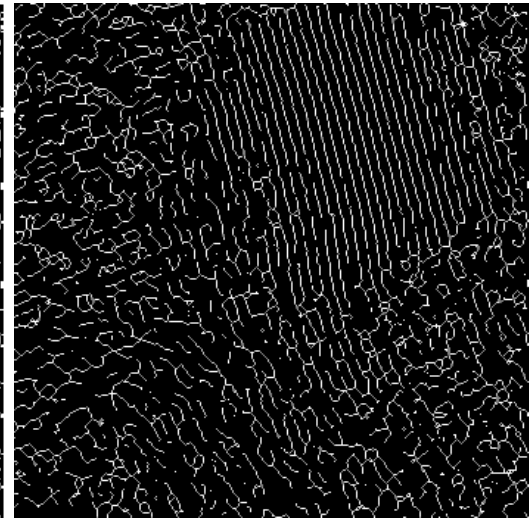

(b) Filtered skeleton

Fig. 2. A skeleton before (a) and after (b) the pruning algorithm.

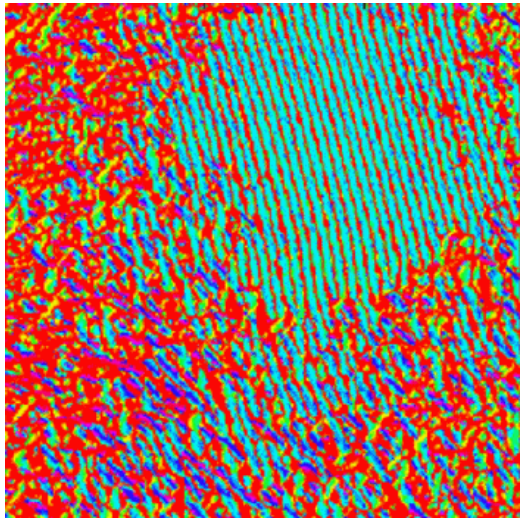

(a) Local orientation

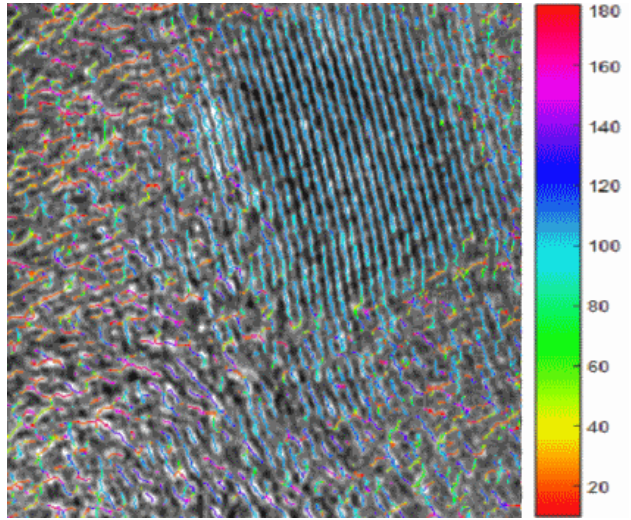

(b) Skeleton orientation

Fig. 3. Application of linear opening to determine the local orientation. (a) local orientation in false colors. (b) combination of the local orientation and the skeleton superimposed on the initial image

\section{Orientation space}

Orientation space has been introduced by Chen and Hsu [3] and popularized later by others authors $[17,8]$. The orientation space is obtained by adding the orientation axis to the image support: it is the result of a transformation consisting in applying rotated copies of an orientation selective filter.

$$
I(x, y) \rightarrow I^{[\theta]}(x, y, \theta)
$$

with $I$ the initial image, $(x, y)$ the coordinates in the initial image, $\theta$ the local orientation, with $\theta \in] 0, \pi]$ and $I^{[\theta]}$ the orientation-space representation of $I$.

It presents several advantages for the evaluation of orientation:

- If two differently-oriented structures intersect in the image, in this space they will belong to different orientation plans, which will ease their distinction.

- The noise spreads over the whole orientation space whereas the signal is located in specific position, which improves the signal-to-noise ratio.

The orientation space allows segmentation of objects even overlapping ones. The segmentation procedure is based on connectivity: pixels belonging to a same object are connected otherwise they 
belong to different objects. However, connected pixels don't always belong to the same object, this is the case for overlapping objects. In order to distinguish overlapping objects, we need a criterion which differs from one object to the other. Local orientation is a way to differentiate touching objects: for two segments to intersect and have the same local orientation is a contradiction.

In order to separate the graphene plans according to their orientation, the skeleton of the picture is put in the orientation space, using the local orientation:

$$
\begin{aligned}
\left.\left.S k^{[\theta]}: D \times\right] 0: \pi\right] & \rightarrow\{0,1\} \\
(x, y, \theta) & \mapsto \begin{cases}1, & \text { if } S k(x, y)=1 \text { and } \theta(x, y)=\theta \\
0, & \text { otherwise }\end{cases}
\end{aligned}
$$

A one dimensional structure $\xi$ in the image will be represented as a one dimensional structure in the orientation space. Moreover a smooth $\xi$ will be connected in this space. Conversely, a continuous but only piece-wise smooth curve will be disconnected in non-differentiable points. Obviously, in order to identify graphene plans, each one should be a unique connected component even if its skeleton is not perfectly smooth. Indeed, a perfectly smooth curve is difficult to obtain due to essentially two reasons: 1 ) we are in a discrete $\mathbb{Z}^{2}$ grid, 2) the local orientation is sampled (taken from a discrete set of angles in eq. 4).

To cope with that issue, a convenient solution is to use a second-generation connectivity [14]. Two structures $\mathrm{X}$ and $\mathrm{Y}$ will be considered connected if they are connected after applying an operator $\psi$ :

$$
X C^{\psi} Y \Leftrightarrow \psi(X) C \psi(Y)
$$

The operator $\psi$ is chosen depending on the way structures are disconnected in the orientation space. We consider two types of imperfection:

1. A noise-corrupted, discontinuous, straight graphene plan is represented in the orientation space as aligned disconnected fragments. One can reconnect these fragments (and only these fragments) using a morphological closing by a line segment oriented in the same direction. Since the orientation of the plans varies this closing also has to vary under translation. However, using the orientation space the spatially varying closing decomposes in a collection of translationinvariant closings. Each subspace $\theta=$ const. contains graphene plans oriented in $\theta$. Hence, for every $\theta=$ const. the closing is a usual translation-invariant closing by a line segment oriented in $\theta$.

To ease the description of the structuring element (here and below in the text) we will use a rotated coordinate system. We will use two unit vectors $u_{\theta \|}$ and $u_{\theta \perp}$ oriented alongside and perpedicularly to the angle $\theta$ of rotation of the system that can be expressed using the initial coordinate system:

$$
\left(\begin{array}{c}
u_{\theta \|} \\
u_{\theta \perp} \\
\theta
\end{array}\right)_{\theta}=\left(\begin{array}{ccc}
\cos (\theta) & -\sin (\theta) & 0 \\
\sin (\theta) & \cos (\theta) & 0 \\
0 & 0 & 1
\end{array}\right)\left(\begin{array}{l}
x \\
y \\
\theta
\end{array}\right)
$$

The coordinates in this new system will be expressed using the notation: $[., ., .]_{\theta}$. Using these coordinates, $[a, b, \theta]_{\theta}$ defines a rectangle $a \times b$ rotated by $\theta$ and located at an altitude $\theta$. Using this new coordinate system, the operator used in the noise tolerant connectivity eq. 7 is a spatially varying closing by a segment:

$$
\varphi_{B_{\theta}} \text { with } B_{\theta}=[d, 0, \theta]_{\theta}
$$

This element is a segment of length $d$ defining the maximum distance between two aligned segments for them to be considered as belonging to the same plan, and $\theta$ gives the translation of the segment used along the $\theta$ axis in the orientation space.

2. Since a discrete skeleton is not always smooth, its segments will distribute over different plans in the orientation space. Moreover, with the discrete angular dimension, there might be 

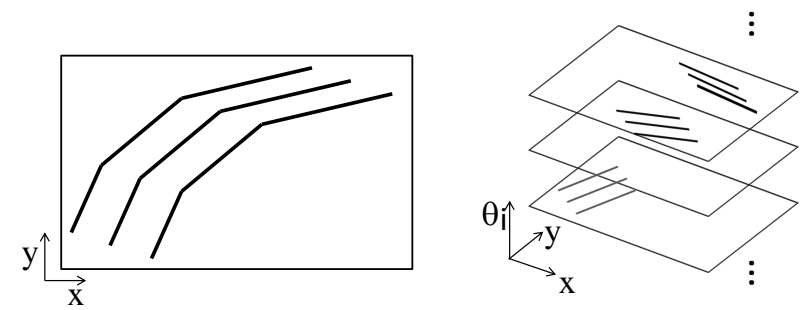

Fig. 4. Curved graphene plans (left) spread over different $\theta_{i}$-subspaces of the orientation space (right).

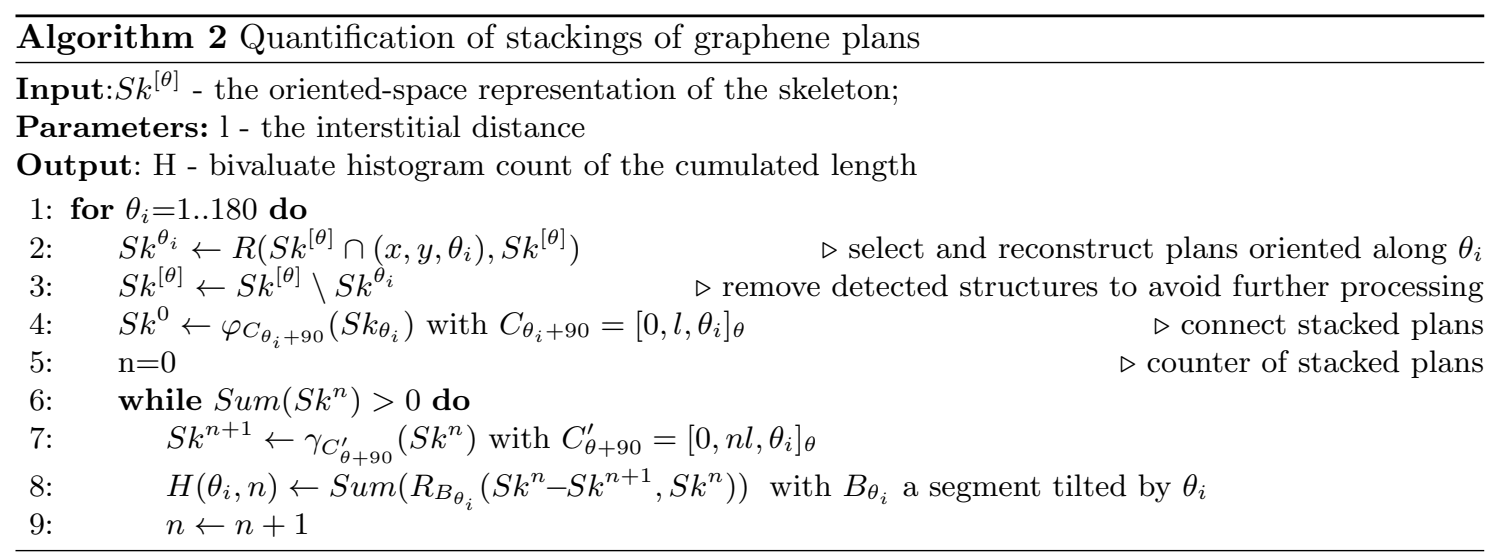

an abrupt change in local orientation. Consequently, even continuous, smooth but tortuous structures will appear in the orientation space as disconnected but have to be identified as one connected component. The operator that allows those curves to be considered as connected is a closing by a vertical segment in the orientation space.

$$
\varphi_{A} \text { with } A=[0,0, h]
$$

The height $h$ of that segment defines the maximum angle between two parts of the curve for which they are still considered to belong to the same structure. When the angle exceeds $h d \theta$, where $d \theta$ is the angular sampling step used in eq. 4 , the two parts are considered as parts of different structures.

\section{Stacking detection}

This study's point is to identify and quantify stackings of plans. Two plans are stacked if: i) they have the same orientation, and ii) the distance between them is the inter-reticular distance, $l$. A stacking is a sequence of two-by-two stacked plans. In the orientation space, structures that are in the same plan and connected after a closing by a segment perpendicular to their orientation of length $l$ are stacked.

The Alg. 2 counts the number of stacked plans. The algorithm must handle the difficulty that even though curved structures spread in the orientation space over several $\theta_{i}$-subspaces (see Fig. 4) their length shouldn't be underevaluated. This is overcome by the use of second-generation connectivity, which allows the detection of a curve as one connected component.

The line 2 detects plans containing at least a portion oriented along $\theta_{i}$ and reconstructs it in its entirety by morphological reconstruction by dilation. $R(x, y)$ is a morphological reconstruction by dilation of $x$ under $y$. See Section 5 below for the structuring element used in the reconstruction. The line 4 detects stackings: plans connected after a closing by $C_{\theta+90}$ belong to the same stack. The lines 6 to 9 measure the cumulated length (using the Sum on the skeleton) using granulometry 
by opening by reconstruction. After, the stackings are quantified using a bivaluate distribution of the cumulated length over orientation and number of stacked plans.

\section{Robustness to noise}

Even organized materials contain imperfections consisting in discontinuous and imperfectly-parallel plans. Such regions wouldn't be detected as stackings in a whole, but as fragmented stacking that would yield biased statistics. Fig. 5 illustrates the situation. To cope with that issue, we set up

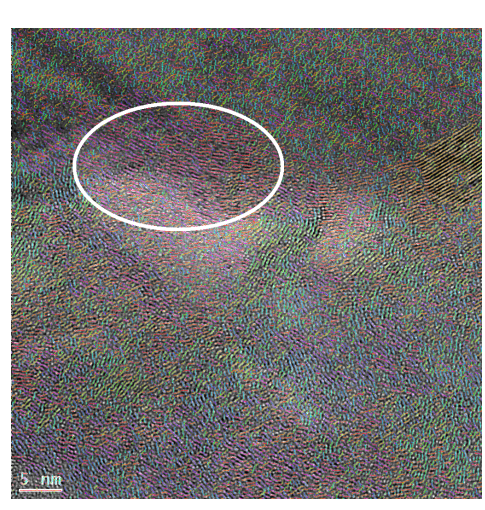

(a) a well-organized zone

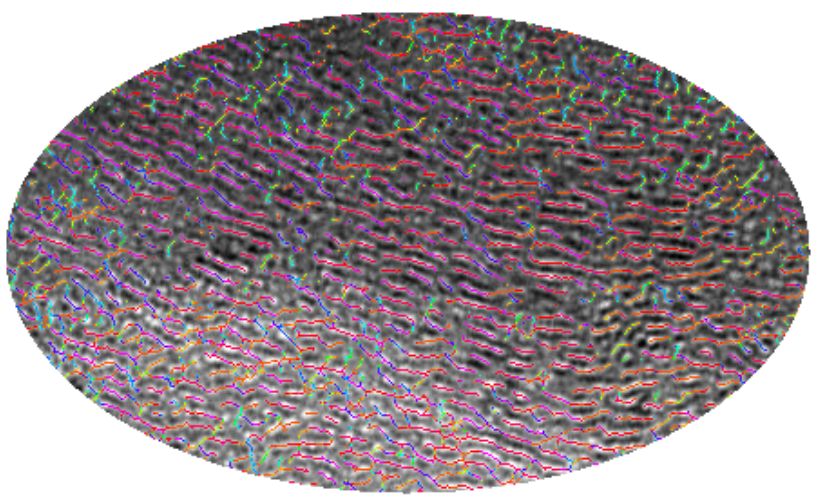

(b) a zoom into (a)

Fig. 5. Zone of interest: a collection of disjoint and imperfectly parallel plans considered as a set of small stacks by the algorithm previously described

tolerances to small discontinuities using second-generation connectivity. As in the previous case, the chosen operator is a closing, but the size of the structuring element has to be adapted to close only admissible imperfections.

The plans are disjoint and not perfectly parallel, so the structuring element needs a length $\mathrm{L}$ along the graphene plans orientation and a height $\mathrm{h}$ along the $\theta$ axis. Moreover, in order to detect stackings as collections of structures separated from an inter-reticular distance, the structuring element needs a thickness of the size of the inter-reticular distance $l$ along the axis perpendicular structures' orientation. The operator used for the tolerances is a spatially varying morphological closing

$$
\varphi_{T_{\theta}} \text { with } T_{\theta}=[L, l, \theta]_{\theta} \times[0,0, h]
$$

with an element $T_{\theta}$ a rectangular parallelepiped $L \times l \times h$ rotated by $\theta$ (obtained as a product of a rotated flat rectangle and a vertically oriented segment). The use of $\varphi_{T_{\theta}}$ before the stacking detection allows the connection of every graphene plans that belong to the same stack. Each stack is then characterized using the previous method.

Fig. 6 illustrates the impact of tolerances on stacking detection. Wider tolerances allow the detection larger stacking zones by gathering disjoint and imperfectly parallel plans. However, wide tolerances also imply the possibility of false detection of stacking in regions where graphene plans are disorganized. A compromise must be found between false and non-detection of stackings.

\section{Results}

The results are presented in the form of a bivariate histogram, presenting the length of the structure given its orientation and the number of stacked plans. 


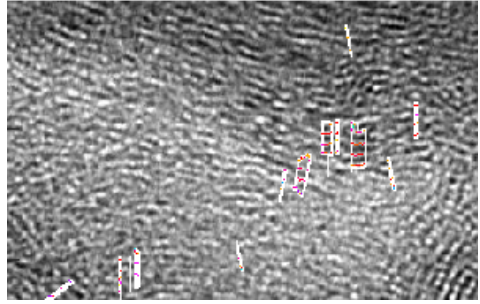

(a) slightly organized without tolerances

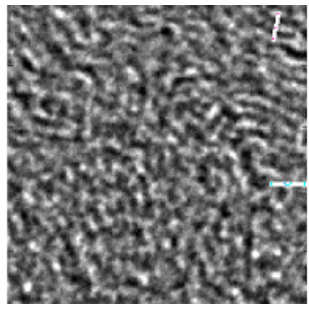

(d) disorganized carbon without (e) tolearnces
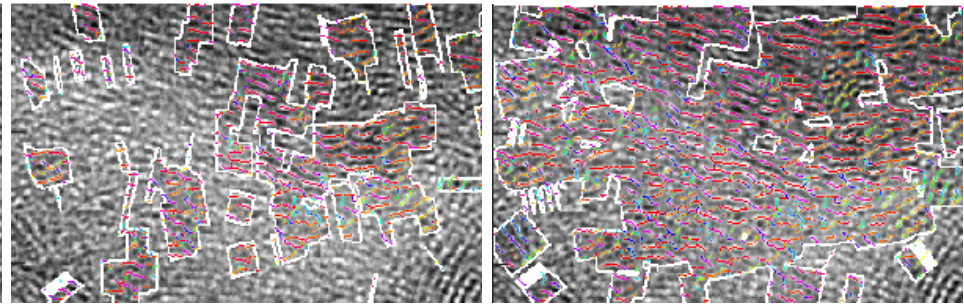

(b) slightly organized carbon with (c) slightly organized carbon with $(L, l, h)=(20,8,3)$

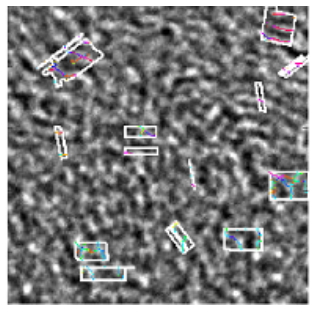

(e) disorganized carbon $(L, l, h)=(20,8,3)$

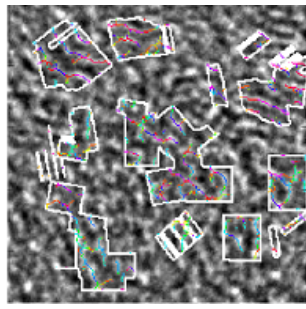

with (f) disorganized carbon with $(L, l, h)=(40,8,4)$
$(L, l, h)=(40,8,4)$

Fig. 6. impact of tolerances on detection of stacks of at least four plans, in organized and disorganized carbon, the detected stacks are highlighted in boxes.

Fig. 7 illustrates the results for organized, slightly organized and disorganized samples. The organized carbon Fig. 7 (a) shows one large stacking oriented around $90^{\circ}$ in the upper-right zone of the image, and several smaller zones oriented around $0^{\circ}$ in the left part of the image. These correspond to the two principal lobes around $90^{\circ}$ and $0^{\circ}$ in the histogram Fig. 7(d). Regarding the lobe around $90^{\circ}$ one can observe that there are up to 30 stacked graphene plans (non-zero values up to 30 of number of stacks in the histogram). The total length histogram axis represents the unnormalized cumulative length of the plans involved in a stacking. The slightly organized carbon, Fig. 7(b), contains a number of small stackings, most oriented roughly horizonally, represented by the lobe around $0^{\circ}$ and $180^{\circ}$ in Fig. 7(e). The disorganized carbon Fig. 7(c) contains almost no graphene stackings but a handful of tiny zones. Its histogram Fig. $7(\mathrm{f})$ is much poorer compared to that of the organized and slightly organized samples.

\section{Conclusion}

This paper presents an original method to identify graphene plans stackings in carbon. For every stacking, it i) determines its orientation, ii) counts the number of stacked plans and iii) estimates the cumulative length of all stacked plans.

These measures can be synthesized in a statistical quantification representing the "organization" rate of a given sample in the form of a bivariate histogram. This is useful for further statistical analysis and comparison of different samples.

This method is fast, simple and intuitive. An adjustable tolerance parameter avoids fractioning due to imperfectly aligned, or discontinuous plans. In this way the stackings identified by this method are closer to what one would intuitively delimit manually as a stacking despite present imperfections.

Experimental results obtained on images taken by an HRTEM microscope are provided to illustrate the validity of the proposed method. 


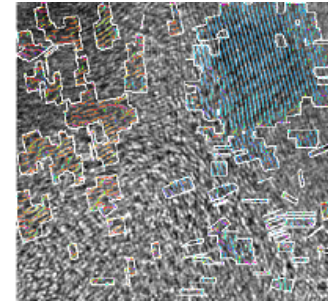

(a) organized carbon

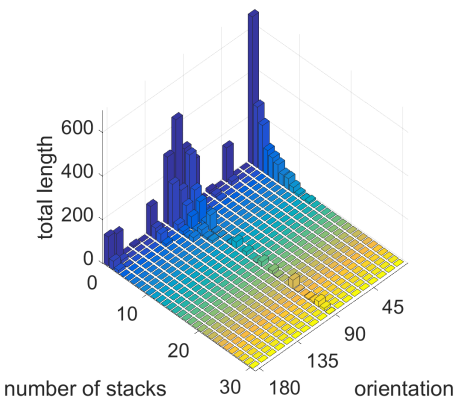

(d) histogram obtained for organised carbon

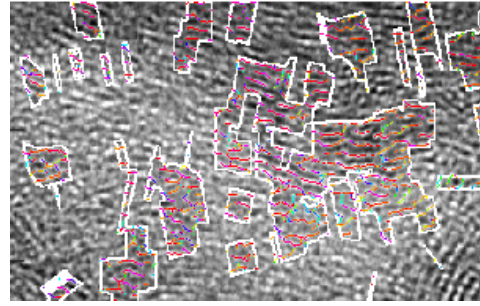

(b) slightly organized carbon

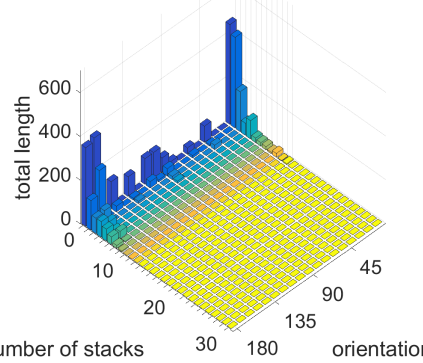

(e) histogram obtained slightly organised carbon

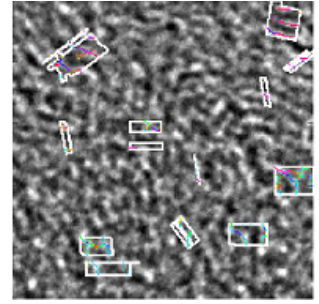

(c) disorganized carbon

Fig. 7. Examples of (a) organized, (b) slightly organized and (c) disorganized carbon, detected stacks are highlighted in boxes. Below, (d-f) the estimated distribution of the length of the stacks depending on the orientation and the number of stacked plans

\section{References}

1. Narottam P Bansal and Jacques Lamon. Ceramic matrix composites: Materials, modeling and technology. John Wiley \& Sons, 2014.

2. E Buet, C Sauder, S Poissonnet, P Brender, R Gadiou, and C Vix-Guterl. Influence of chemical and physical properties of the last generation of silicon carbide fibres on the mechanical behaviour of sic/sic composite. Journal of the European Ceramic Society, 32(3):547-557, 2012.

3. Y.S. Chen and W.H. Hsu. An interpretive model of line continuation in human visual perception. Patern Recognition, 22(5):619-639, 1989.

4. JP Da Costa, P Weisbecker, B Farbos, J-M Leyssale, GL Vignoles, and C Germain. Investigating carbon materials nanostructure using image orientation statistics. Carbon, 84:160-173, 2015.

5. B Farbos, P Weisbecker, HE Fischer, J-P Da Costa, M Lalanne, G Chollon, C Germain, GL Vignoles, and J-M Leyssale. Nanoscale structure and texture of highly anisotropic pyrocarbons revisited with transmission electron microscopy, image processing, neutron diffraction and atomistic modeling. Carbon, 80:472-489, 2014.

6. Yutai Katoh, Lance Lewis Snead, Charles H Henager, Akira Hasegawa, Akira Kohyama, Bruno Riccardi, and Hans Hegeman. Current status and critical issues for development of sic composites for fusion applications. Journal of Nuclear Materials, 367:659-671, 2007.

7. J-M Leyssale, J-P Da Costa, Christian Germain, Patrick Weisbecker, and GL Vignoles. Structural features of pyrocarbon atomistic models constructed from transmission electron microscopy images. Carbon, 50(12):4388-4400, 2012.

8. Miguel A. Luengo-Oroz. Spatially-variant structuring elements inspired by the neurogeometry of the visual cortex. In P. Soille, M. Pesaresi, and G. K. Ouzounis, editors, Mathematical Morphology and Its Applications to Image and Signal Processing. Springer Berlin Heidelberg, 2011.

9. Roger R Naslain. The design of the fibre-matrix interfacial zone in ceramic matrix composites. Composites Part A: Applied Science and Manufacturing, 29(9):1145-1155, 1998.

10. N. Otsu. A threshold selection method from gray-level histograms. IEEE Transactions on Systeme, Man and Cybernetics, 1979.

11. Pascaline Pré, Guillaume Huchet, Dominique Jeulin, Jean-Noël Rouzaud, Mohamed Sennour, and Alain Thorel. A new approach to characterize the nanostructure of activated carbons from math- 
ematical morphology applied to high resolution transmission electron microscopy images. Carbon, 52:239-258, 2013.

12. B Reznik and KJ Hüttinger. On the terminology for pyrolytic carbon. Carbon, 40(4):621-624, 2002.

13. Jean-Noël Rouzaud and Christian Clinard. Quantitative high-resolution transmission electron microscopy: a promising tool for carbon materials characterization. Fuel Processing Technology, 77:229 $235,2002$.

14. J. Serra. Connectivity on complete lattices. Journal of Mathematical Imaging and Vision, 9(3):231$251,1998$.

15. Hong-Shig Shim, Robert H Hurt, and Nancy YC Yang. A methodology for analysis of 002 lattice fringe images and its application to combustion-derived carbons. Carbon, 38(1):29-45, 2000.

16. Pal Toth, Arpad B Palotas, Eric G Eddings, Ross T Whitaker, and JoAnn S Lighty. A novel framework for the quantitative analysis of high resolution transmission electron micrographs of soot i. improved measurement of interlayer spacing. Combustion and Flame, 160(5):909-919, 2013.

17. M. Van Ginkel, L. J. Van Vliet, and P. W. Verbeek. Applications of image analysis in orientation space. In Fourth Quinquennial Review 1996-2001 Dutch Society for Pattern Recognition and Image Processing. NVPHBV, 2001.

18. Randy L Vander Wal, Aaron J Tomasek, Michael I Pamphlet, Christina D Taylor, and William K Thompson. Analysis of hrtem images for carbon nanostructure quantification. Journal of Nanoparticle Research, 6(6):555-568, 2004.

19. Kuen Yehliu, Randy L Vander Wal, and André L Boehman. Development of an hrtem image analysis method to quantify carbon nanostructure. Combustion and Flame, 158(9):1837-1851, 2011.

20. Richard W. Hall Zichen Guo. Parallel thinning with two-subiteration algorithms. Communications of the ACM, 32(3):359-373, 1989. 